\title{
Development of Microwave Kinetic Inductance Detectors for IR single photon counting
}

\author{
R. Mezzena ${ }^{1,2} \cdot$ M. Faverzani $^{3,4} \cdot$ E. Ferri ${ }^{3,4} \cdot$ A.

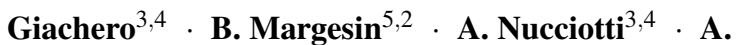 \\ Puiu $^{3,4}$. A. Vinante ${ }^{6,7}$
}

the date of receipt and acceptance should be inserted later

\begin{abstract}
We have developed Microwave Kinetic Inductance Detectors suitable for nearIR single photon counting. Our films are made of titanium and titanium nitride, deposited in a multi-layer structure Ti/TiN/Ti/TiN with a total thickness of $44 \mathrm{~nm}$. The film has a transition temperature of $1.2 \mathrm{~K}$ and a surface kinetic inductance of $34 \mathrm{pH} / \mathrm{sq}$. The resonator was designed with lumped elements and consists of two blocks of interdigitated capacitors connected by a meandered stripe inductor. The resonator resonance frequency is $6.8 \mathrm{GHz}$ and the internal quality factor is 125000 . The detector is read out with the usual homodyne scheme and calibrated with light pulses produced by a laser diode with wavelength 1550 $\mathrm{nm}$. For the 0- and 1-photon peak we measure a FWHM energy resolution of $0.44 \mathrm{eV}$ and $0.56 \mathrm{eV}$ respectively. This resolution is sufficient to resolve events with up to 4 photons.
\end{abstract}

Keywords Microwave Kinetic Inductance Detectors, Single photon detector, Ti/TiN multilayer films

\section{Introduction}

Microwave Kinetic Inductance Detectors MKIDs have aroused great interest since its appearance [1] particularly with regards to the capability to build large sensor arrays. A considerable effort has been devoted to prove theoretically and experimentally that MKIDs can compete with the most sensitive low temperature sensors detecting electromagnetic radiation ranging from the $\mathrm{mm}$ waves to the $\mathrm{X}$ ray [2]. At $1550 \mathrm{~nm}$ single photon number resolving capability has been proved but the energy resolution experimentally demonstrated is still one order of magnitude far from the theoretical limits [5, 6]. Therefore, it is reasonable to

\section{R. Mezzena}

E-mail: renato.mezzena@unitn.it

${ }^{1}$ Dipartimento di Fisica, Università di Trento, I-38123 Povo, Trento, Italy

2 INFN - TIFPA Trento Institute for Fundamentals Physics Applications, I-38123, Trento, Italy

${ }^{3}$ Dipartimento di Fisica, Università di Milano-Bicocca, Milano I-20126, Italy

${ }^{4}$ INFN Sezione di Milano Bicocca, Milano I-20126, Italy

${ }^{5}$ Fondazione Bruno Kessler (FBK), I-38123 Povo, Trento, Italy

${ }^{6}$ Istituto di Fotonica e Nanotecnologie, CNR and FBK, I-38123 Povo, Trento, Italy

${ }^{7}$ Department of Physics and Astronomy, University of Southampton, SO17 1BJ, United Kingdom 
push for further improvements. In order to approach this target, we have focused our research on the film material and the resonator layout. In particular we have carried out an extensive investigation [3] on Ti/TiN multilayer films aiming to achieve the control of the superconducting and electrical properties important for MKIDs. In this paper we describe our preliminary results and we show that the detector can resolve single photon number. The resolution obtained is within a factor two from the best value published in literature [6]. Compared to the latter work the main difference of our design is a larger detector sensitive volume. We are confident to achieve better results reducing the volume by a factor of 10 . In addition, we plan to decrease the superconductor energy gap by lowering the film transition temperature.

\section{Detector}

2.1 Resonator design and measured features

We have simulated and experimentally studied two main resonator layouts : one with distributed and one with lumped capacitance $C$ and inductance $L$. The first kind, is similar to

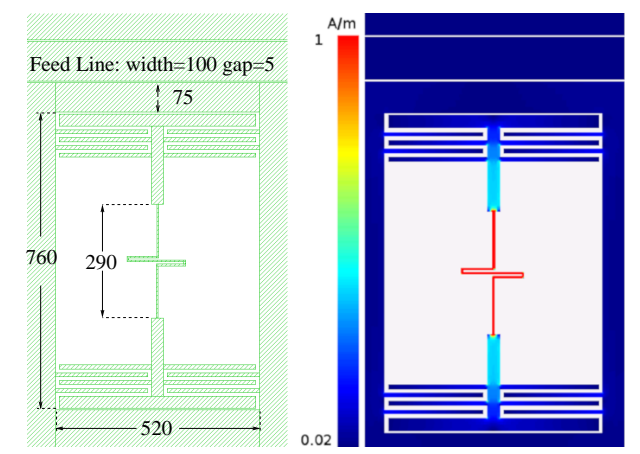

Fig. 1 Left: Resonator layout; sizes are in $\mu \mathrm{m}$. The interdigitated capacitors gap is $10 \mu \mathrm{m}$; the inductor line width changes from $30 \mu \mathrm{m}$ in the largest part to $5 \mu \mathrm{m}$ in the central narrow and folded part. Right: The simulated current density at resonance frequency (Color figure online).

that reported in ref [4] but has larger volume, in the range $1400-3000 \mu \mathrm{m}^{3}$. With this layout we have obtained resonators with quite high internal quality factor in the range $4 \times 10^{5}-1 \times 10^{6}$, but due to the large volumes, we were not able to achieve an energy sensitivity suitable for single photon counting. The layout with lumped $(L, C)$ elements reported in Fig. 1 has made possible better performances. It consists of two blocks of interdigitated capacitors connected by an inductive element whose central part is narrower, having a line width of $5 \mu \mathrm{m}$. The resonator and the CPW feed line are separated by a ground area which determines the coupling between the two. At $20 \mathrm{mK}$ we have measured a resonance frequency $v_{r}=6.802 \mathrm{GHz}$, an internal quality factor $Q_{i}=125000$, a coupling quality factor $Q_{c}=27170$ and a total quality factor $Q_{t}=22300$. The resonator was previously simulated with the Sonnet software in order to predict the resonance frequency and the coupling with the feed line. Also the current density was simulated and the result at the resonance frequency is reported in Fig. 1. Since the most sensitive detector part is that with the highest 
current density, the effective volume of the detector is essentially limited to the inductor central part which corresponds to $128 \mu \mathrm{m}^{3}$.

\subsection{Fabrication}

Our detectors are made out of a thin film of titanium and titanium nitride, deposited in a multi-layer structure Ti/TiN/Ti/TiN. This choice allows to optimize the kinetic inductance $L_{k}$ and the superconductor energy gap $\Delta_{0}$. The detector responsivity depends crucially on these parameters [2]. High $L_{k}$ values can be obtained due to the high resistivity in the normal state of TiN. $\Delta_{0}$ depends on the superconducting transition temperature $T_{c}$. Thanks to the proximity effect, we can tune $T_{c}$ by properly choosing the thickness of the Ti and TiN layers [3]. In our film the Ti and TiN single layer thickness are 10 and $12 \mathrm{~nm}$ respectively and the total thickness is $44 \mathrm{~nm}$. This results in a normal state resistivity of $131 \mu \Omega \mathrm{cm}$, a critical temperature $T_{c}=1.21 \mathrm{~K}$ and a surface kinetic inductance of $34 \mathrm{pH} / \mathrm{sq}$. The films have been fabricated by sputter deposition of Ti/TiN onto p-type, $<100>$, high resistive FZ, 6" silicon wafers with a resistivity larger than $5000 \Omega \mathrm{cm}$. The films were deposited with an Eclipse ${ }^{\circledR}$ Mark II $^{\mathrm{TM}}$ sputtering system using a titanium target in combination with reactive sputtering with nitrogen. All films were deposited at $350^{\circ} \mathrm{C}$. The $10 \mathrm{~nm}$ thick Ti layers were deposited in $8 \mathrm{sec}$ with $1 \mathrm{~kW}$ RF power applied. The TiN layers were deposited with $6 \mathrm{~kW}$ RF power, $200 \mathrm{~V}$ bias applied and an argon flow of $30 \mathrm{sccm}$ and an nitrogen flow of $45 \mathrm{sccm}$.

\subsection{Readout}

The experimental set-up is shown in Fig. 2. The MKID is placed inside a copper box which is attached to the Mixing Chamber stage of a cryogen-free dilution refrigerator. A range of temperature from 20 to $150 \mathrm{mK}$ has been explored, the best result were obtained at $110 \mathrm{mK}$. The signal is read out employing an homodyne detection scheme. Some components are critical in order to avoid spoiling the detector sensitivity. We use a cryogenic HEMT preamplifier anchored to a $3.8 \mathrm{~K}$ stage, with a noise temperature of $4 \mathrm{~K}$ and a gain of $33 \mathrm{~dB}$. A $20 \mathrm{~dB}$ attenuator is placed at the $3.8 \mathrm{~K}$ stage and another one at the mixing chamber stage in order to suppress the thermal noise coming from the room temperature electronics. We estimate that the room temperature components contribute to an increase by $6 \%$ of the overall noise temperature of the system. The power of the microwave excitation signal is set $1 \mathrm{~dB}$ just below the onset of nonlinearities in the resonator. The MKID output signal is connected to the cryogenic preamplifier by means of a superconducting coaxial cable. After further room temperature amplification the signal is fed into a IQ mixer and passband-filtered in the band $10-300 \mathrm{kHz}$. The low frequency cutoff reduces the noise contributed by the two level system affecting the superconducting resonators[5]. A phase shifter is used in order to compensate the delays between local and the RF mixer inputs due to different cable length and interposed devices. Moreover a fine tuning of the phase shifter permits to align the pulse signal, produced when the MKID is irradiated, into the mixer I or Q port.

The optical response is investigated irradiating the MKID with light pulses produced by a laser diode with wavelength $1550 \mathrm{~nm}$ and $6 \mathrm{~mW} \mathrm{cw}$ power. The laser temperature is controlled with a stability better than $0.1^{\circ} \mathrm{C}$. The laser is driven by a pulse generator with repetition frequency of $33 \mathrm{~Hz}$. A $35 \mathrm{~dB}$ optical attenuator is inserted between the laser and the $125 \mu$ m diameter single mode optical fiber which guides the light inside the refrigerator cryostat. The optical fiber end which irradiates the detector is fixed about $2 \mathrm{~mm}$ above the 


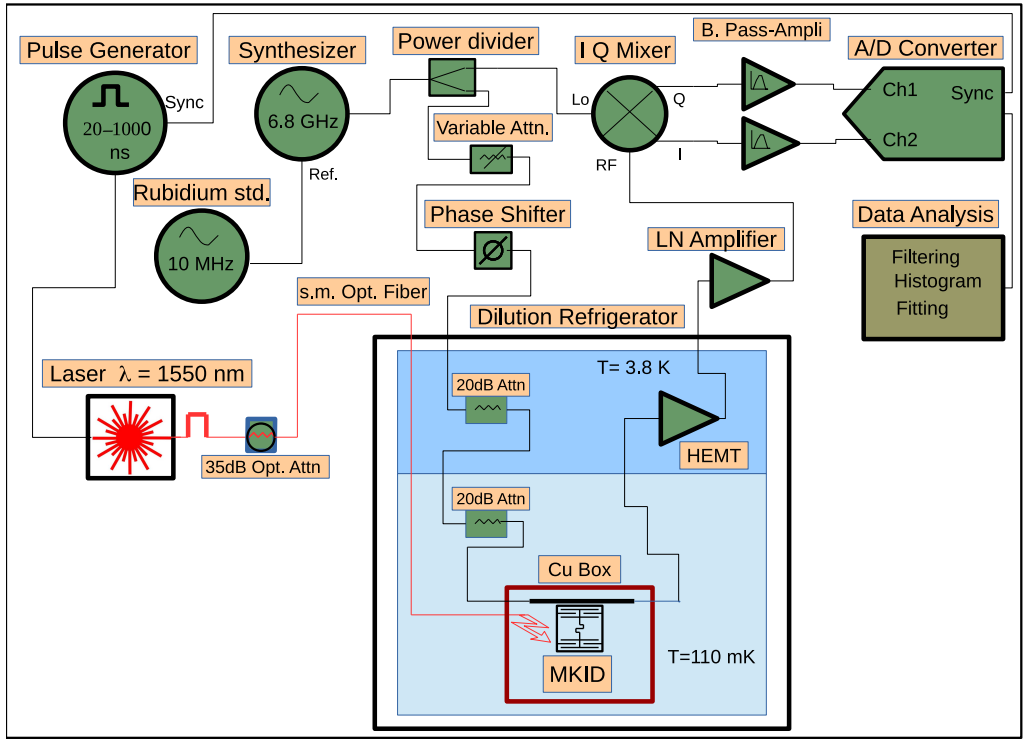

Fig. 2 Scheme of the experimental setup (Color figure online.)

chip. The present setup is not explicitally optimized for a maximization of the coupling efficiency.

Since it is well proved $[5,6]$ that frequency quadrature read out ensures a better signal to noise ratio we collect only the $\mathrm{Q}$ output of the mixer. As we are operating in a small signal linear regime, the Q output can linearly reproduce the optical pulses. In order to test the detector linearity, we have generated optical pulses with time widths in the range $200-800 \mathrm{~ns}$. The pulse time width is directly proportional to the pulse energy, i.e. the mean photon number per pulse. In Fig. 3 (left) the averaged pulse height collected from the Q mixer output is plotted as a function of pulse time width. The data linear regression is shown and confirms the assumption of linearity for the selected range of pulse energy. In Fig. 3 (right) we report the pulse response whose decay time is $75 \mu \mathrm{s}$.
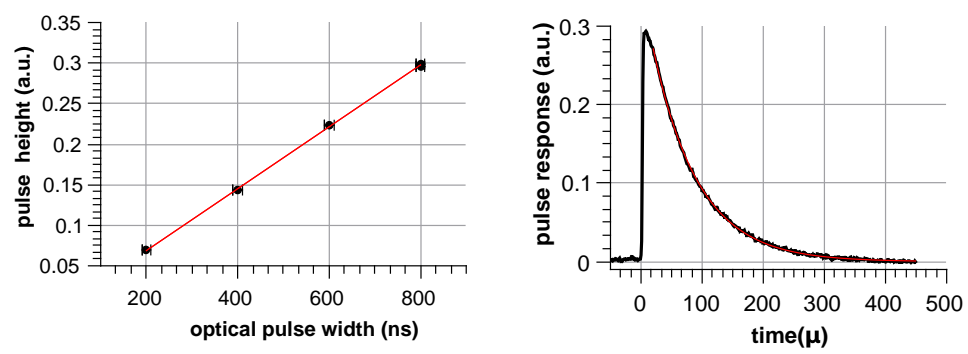

Fig. 3 Left: The detector averaged pulse height as a function of the optical pulse width. The red curve is the linear regression: it confirms the linearity between absorbed energy and pulse response. Right: The averaged pulse response. The red curve is the exponential regression of the pulse decay interval. (Color figure online.) 


\section{Results and discussion}

The data sampling and the subsequent analysis adopts the following procedure. The pulse generator is set with a pulse width in the range $200-800 \mathrm{~ns}$ in order to send a specific average numbers of photons to the detector. A 16 bit A/D acquisition card synchronized with the pulse generator, samples at $12.5 \mathrm{MHz}$ a total number $N_{p}$ of pulses, where we set $10000 \leq N_{p} \leq 50000$. A matched filter is applied to the stored raw data in order to improve the signal to noise ratio. Such filter requires a pulse template which is estimated by separately averaging 10000 optical pulses with $1000 \mathrm{~ns}$ width. A histogram of the amplitude $x$ of the reconstructed pulses is then produced. Depending on the average numbers of photons absorbed by the detector, a fitting procedure with a proper sum of $N$ gaussian functions convolved with a Poisson distribution

$$
\sum_{n=0}^{N} a \frac{\lambda^{n}}{n !} \exp \left(\frac{(x-n b)^{2}}{2 \sigma_{n}^{\prime 2}}\right)
$$

with $a, \lambda, \sigma_{n}^{\prime}$, and $b$ free parameters is performed. Here $a$ is a normalization parameter, $\lambda$ is the average number of events, $\sigma_{n}^{\prime}$ is the standard deviation of the $n$th gaussian peaks, and $b$ is the calibration factor. In order to calibrate the histogram abscissa axis in photon numbers, the amplitude values $x$ are multiplied by the inverse of the $b$ factor obtained by the fitting procedure. The standard deviation of the $n$th gaussian peak expressed in photon number is $\sigma_{n}=\sigma_{n}^{\prime} / b$ and the fit parameter $\lambda$ coincides with the mean number of photons absorbed per pulse. In the two plots of Fig. 4 we report the pulse height histogram obtained with mean
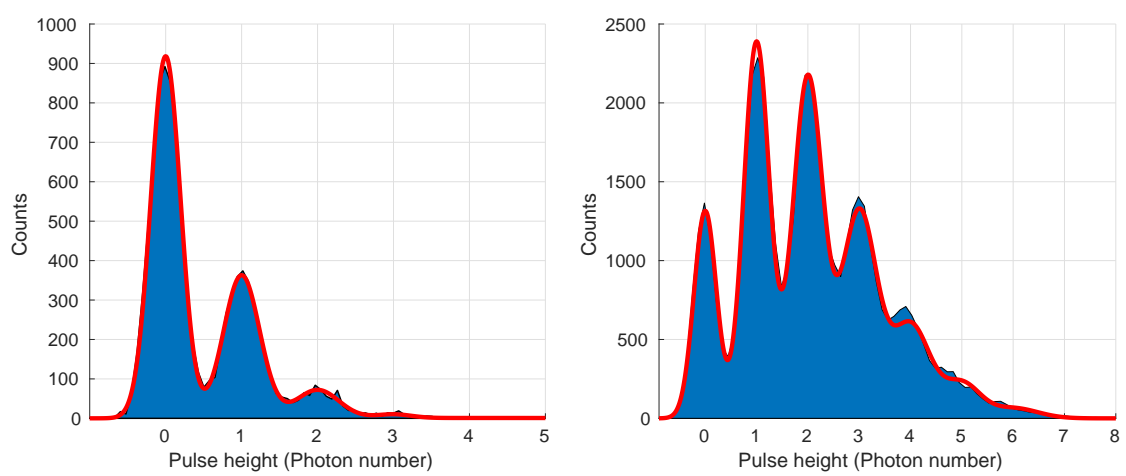

Fig. 4 Left: Histogram of 10000 pulse heights calibrated in photon numbers; the mean photon number is $\lambda=0.40$ Right: Histogram of 50000 pulse heights; the mean photon number is $\lambda=1.81$. The red curves are obtained from the the best fits of Eq. 1 to the data, after applying the calibration factor (Color figure online.)

photon number $\lambda=0.40$ and $\lambda=1.81$ respectively. We are able to resolve events with up to 4 photons. Indeed, the standard deviation $\sigma_{n}$ increases with $n$ as shown in Fig. 5 and peaks with $\sigma_{n} \gtrsim 0.4$ are not distinguishable. The broadening of peaks with $n \geq 1$ can be related to the non uniform responsivity of the detector absorbing area. In this case, we expect a broadening increasing linearly with $n$, which is consistent with the trend observed in Fig. 5. At $1550 \mathrm{~nm}$ the energy of a single photon is $h v=0.8 \mathrm{eV}$. Converting $\sigma_{n}$ in energy, for the 0 - and 1-photon peak we obtained a FWHM energy resolution of $0.44 \mathrm{eV}$ and $0.56 \mathrm{eV}$ respectively. 

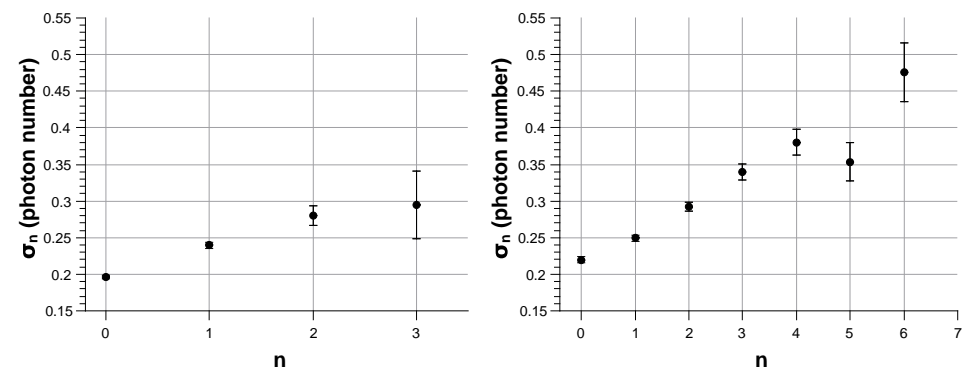

Fig. 5 The standard deviation $\sigma_{n}$ of the $n$th resolved peak is plotted for $\lambda=0.4$ in the Left and for $\lambda=1.81$ in the Right plot.

\section{Conclusion}

We have employed the Ti/TiN multilayer film technology to develop MKIDs capable to resolve single photons at $1550 \mathrm{~nm}$. For the 0 - and 1-photon peak we obtained a FWHM energy resolution of $0.44 \mathrm{eV}$ and $0.56 \mathrm{eV}$ respectively; events up to 4 photons can be resolved. This result is within a factor of two from the best value published in literature. As proved in ref. [6] the energy resolution improves by reducing the volume of the resonator inductor. Therefore in the next design we aim at a further improvement by reducing the inductor volume by about a factor of 10 . We also plan to reduce the film critical temperature below $1 \mathrm{~K}$ in order to decrease the superconductor energy gap.

We have not addressed the detection efficiency optimization. We consider MKIDs as PNR detector since the pulse response is proportional to the number of photons absorbed. This was proved with a statistical method: further investigations are needed to accomplish a more stringent fidelity criterion [7] which requires a detection efficiency close to $100 \%$.

Acknowledgements This work is carried out in the framework of the KIDS R\&D project funded by the Istituto Nazionale di Fisica Nucleare (INFN), Italy in the Commissione Scientifica Nazionale 5 (CSN5). We acknowledge G. Fontana for his valuable support.

\section{References}

1. P.K. Day, H.G. LeDuc, B.A. Mazin, A. Vayonakis and J. Zmuidzinas Nature 425, 817, (2003), DOI:10.1038/nature02037

2. J. Zmuidzinas Annu. Rev. Condens. Matter Phys. 3, 169, (2012), DOI:10.1146/annurevconmatphys-020911-125022

3. A. Giachero, P.Day, P. Falferi, M. Faverzani, E. Ferri, C. Giordano, B. Margesin, F. Mattedi, R. Mezzena, R. Nizzolo, A. Nucciotti, J. Low Temp. Phys. 176, 155, (2014), DOI:10.1007/s 10909-013-1078-0

4. B.A. Mazin, B. Bumble, S.R. Meeker, K. O’Brien, S. McHugh and E. Langman, Opt. Express 20,1503, (2012), DOI:10.1364/OE.20.001503

5. J. Gao, M.R. Vissers, M.O. Sandberg, F.C.S. da Silva, S.W. Nam, D.P. Pappas, D.S. Wisbey, E.C. Langman, S.R. Meeker, B.A. Mazin, H.G. Leduc, J. Zmuidzinas, and K. D. Irwin, Appl. Phys. Lett. 101, 142602, (2012), DOI:10.1063/1.4756916. 
6. W.Guo, X.Liu, Y.Wang, Q.Wei, L. F.Wei, J.Hubmayr, J.Fowler, J.Ullom, L.Vale, M. R.Vissers and J.Gao, Appl. Phys. Lett. 110, 212601 , (2017), DOI:10.1063/1.4984134

7. M. Jönsson and G. Björk, Phys. Rev. A 99, 043822, (2019), DOI:10.1103/PhysRevA.99.043822 